Pacific

Journal of

Mathematics

UNIQUENESS THEOREM FOR ORDINARY DIFFERENTIAL EQUATIONS WITH HÖLDER CONTINUITY

YifEI PAN, MeI WANG AND Yu YAN 


\title{
UNIQUENESS THEOREM FOR ORDINARY DIFFERENTIAL EQUATIONS WITH HÖLDER CONTINUITY
}

\author{
YIFEI PAN, MEI WANG AND YU YAN
}

\begin{abstract}
We study ordinary differential equations of the type $u^{(n)}(t)=f(u(t))$, with initial conditions $u(0)=u^{\prime}(0)=\cdots=u^{(m-1)}(0)=0$ and $u^{(m)}(0) \neq 0$, where $m \geq n$; no additional assumption is made on $f$. We establish some uniqueness results and show that $f$ is always Hölder continuous.
\end{abstract}

\section{Introduction}

The question of finding criteria for the uniqueness of solutions has been a constant theme in the study of ordinary differential equations for a very long time, and a wealth of results have been established. The one most quoted in textbooks is perhaps the Lipschitz uniqueness theorem, which states that in the equation $y^{(n)}(x)=f\left(x, y, y^{\prime}, \ldots, y^{(n-1)}\right)$, if the function $f\left(x, z_{1}, z_{2}, \ldots, z_{n}\right)$ is Lipschitz continuous with respect to $z_{1}, z_{2}, \ldots, z_{n}$, then the initial value problem has a unique local solution. Generally speaking, to ensure the uniqueness of solutions to an ODE, we need to assume some condition on the function $f$ besides continuity, the Lipschitz condition being one example. Most of the research in this topic has been devoted to finding the appropriate condition, and there are many nice results, such as the classical theorems by Peano, Osgood, Montel and Tonelli, and Nagumo. An extensive and systematic treatment of the available results is provided in [Agarwal and Lakshmikantham 1993].

In this paper, we approach the uniqueness problem from a different perspective and relate it to the unique continuation problem. We study autonomous ODEs of the type $u^{(n)}(t)=f(u(t))$, where $u \in C^{\infty}([0,1])$ and no additional assumption is made on the function $f$.

If we assume the initial conditions $u(0)=u^{\prime}(0)=\cdots=u^{(n-1)}(0)=0$, the solution is not unique. The following is a trivial example.

Example 1. $u(t)=t^{3}$ satisfies $u^{\prime \prime}(t)=6 u^{1 / 3}$ and $u(0)=u^{\prime}(0)=0$. Another solution to this initial value problem is $u \equiv 0$.

The research of Yu Yan was partially supported by the Scholar in Residence program at Indiana University-Purdue University Fort Wayne.

MSC2010: 34A12.

Keywords: uniqueness of solutions, ordinary differential equations. 
It is no surprise that uniqueness fails in this example, because the function $f(u)=6 u^{1 / 3}$ has fairly strong singularity at 0 . From another perspective, this example shows that if a solution and its derivatives up to order $n-1$ all vanish at 0 , it is not guaranteed to be the zero function. On the other hand, even if all its derivatives vanish at 0 , the solution still may not be identically 0 .

Example 2. The function

$$
u(t)= \begin{cases}e^{-1 / t} & 0<t \leq 1 \\ 0 & t=0\end{cases}
$$

is in $C^{\infty}([0,1])$, and

$$
u^{(k)}(0)=0 \quad \text { for all } \quad k \in \mathbb{N} \text {. }
$$

Let

$$
f(s)= \begin{cases}s(\ln s)^{2} & s>0 \\ 0 & s=0 .\end{cases}
$$

Then $u(t)$ satisfies the equation

$$
u^{\prime}=f(u) .
$$

However, this equation has another solution, $u \equiv 0$, which also satisfies (1).

This function $u(t)$ is also a classical example in the study of the unique continuation problem, which asks when we can conclude that a function is locally identically zero if its derivatives all vanish at a point. Here is one result in this line:

Theorem 1.1 [Pan and Wang 2008]. Let $g(x) \in C^{\infty}([a, b]), 0 \in[a, b]$, and

$$
\left|g^{(n)}(x)\right| \leq C \sum_{k=0}^{n-1} \frac{\left|g^{(k)}(x)\right|}{|x|^{n-k}}, \quad x \in[a, b]
$$

for some constant $C$ and some $n \geq 1$. Then

$$
g^{(k)}(0)=0 \quad \text { for all } \quad k \geq 0
$$

implies

$$
g \equiv 0 \quad \text { on } \quad[a, b] .
$$

The order of singularity of $|x|$ at 0 in (2), that is, $n-k$, is sharp, as one example in [Pan and Wang 2008] shows. This theorem is crucial to the proof of our main theorem below.

The previous two examples suggest that to guarantee uniqueness near 0 , the solution needs to vanish to sufficiently high order, but not to the infinite order. So we assume that it satisfies the initial conditions $u(0)=u^{\prime}(0)=\cdots=u^{(m-1)}(0)=0$ and $u^{(m)}(0)=a \neq 0$, where $m \geq n$. That is, the order of the lowest nonvanishing derivative of $u$ at 0 is no less than the order of the equation. From the equation 
it is not difficult to see that $f$ is differentiable away from 0 ; however, it is not differentiable at 0 , as shown by Example 1 with $m=3$.

Due to the lack of information about the regularity of $f$, the available uniqueness theory no longer applies to this type of equation. We will show that because $u$ has sufficiently high vanishing order at 0 , such solutions are unique near 0 . Specifically, we have the following result.

Theorem 1.2. Let $u(t) \in C^{\infty}([0,1))$ be a solution of the differential equation

$$
u^{(n)}(t)=f(u(t))
$$

where $n \geq 1$ and $f$ is a function. Assume that $u$ satisfies

$$
u(0)=u^{\prime}(0)=\cdots=u^{(m-1)}(0)=0 \quad \text { and } \quad u^{(m)}(0)=a \neq 0,
$$

with $m \geq n$. Then such a solution $u(t)$ is unique for $t$ near 0 .

The proof of Theorem 1.2 is carried out in two steps. First, we show the following result concerning the derivatives of $u$ at 0 .

Lemma 1.3. Let $u(t)$ be a solution that satisfies Equations (3) and (4). The derivative of $u$ at 0 of any order equal to or higher than $m$, that is, $u^{(k)}(0)$ for any $k \geq m$, depends only on $m, n$, and the behavior of the function $f$ near 0 .

In the second step, suppose there are two solutions $u$ and $v$, both satisfying (3) and (4); then by Lemma 1.3, the function $u(t)-v(t)$ and all its derivatives vanish at 0 . Making use of Theorem 1.1, we can show that $u-v \equiv 0$.

Typically, for an $n$-th order ODE, we need only $n$ initial conditions. Theorem 1.1 shows that in some sense, the lack of information about $f$ can be compensated by assuming additional derivative information at the initial point.

Interestingly, it turns out that the solution is unique as long as the vanishing order is no less than the order of the equation, but the actual vanishing order and the value of the lowest nonzero derivative are not essential.

Theorem 1.4. Suppose $u_{1}$ and $u_{2}$ are two solutions of (3) that satisfy

$$
u_{1}(0)=u_{1}^{\prime}(0)=\cdots=u_{1}^{(m-1)}(0)=0, \quad u_{1}^{(m)}(0)=a \neq 0
$$

and

$$
u_{2}(0)=u_{2}^{\prime}(0)=\cdots=u_{2}^{(l-1)}(0)=0, \quad u_{2}^{(l)}(0)=b \neq 0,
$$

where $m, l \geq n$. Then $m=l, a=b$, and $u_{1} \equiv u_{2}$ for small $t$.

The proof of Lemma 1.3 will be given in Section 2, and the proofs of Theorems 1.2 and 1.4 will be given in Section 3 .

Naturally, we would like to ask if the result still holds if one of the solutions in Theorem 1.4 has vanishing order lower than $n$, the order of the equation. 
Conjecture. Suppose (3) has a solution $u(t)$ that satisfies (4) with $m \geq n$. Then it cannot possess another solution $v(t)$ that satisfies initial conditions

$$
v(0)=v^{\prime}(0)=\cdots=v^{(l-1)}(0)=0 \quad \text { and } \quad v^{(l)}(0)=b \neq 0,
$$

where $l<n$.

We can show that this conjecture is true if $m=n+1$ or $l$ and $m$ are relatively prime. However, there are some difficulties in the general case and we have not been able to prove the full conjecture.

Although in Theorems 1.2 and 1.4 we do not need to make any assumptions about the function $f$, we can actually obtain interesting information about it. Suppose there is a function $u(t)$ that satisfies Condition (4); then as shown in Section 2, locally $t$ can be expressed as a function of $u$, and therefore we can express $u^{(n)}(t)$ locally as a function $f$ of $u$, so $u^{(n)}(t)=f(u)$. The next theorem shows that the function $f$ is Hölder continuous in an interval $[0, \delta]$ for small $\delta>0$.

Theorem 1.5. Suppose a function $u(t)$ satisfies Condition (4); then Equation (3) holds for some function $f$, where $m \geq n$ and there is a constant $\delta>0$ such that $f$ is uniformly Hölder continuous in the interval $[0, \delta]$.

This theorem is proved after Theorem 1.4 in Section 3.

A summary of Theorems 1.2 and 1.5 is that any smooth function of finite order vanishing at 0 is a unique local solution of a differential equation in the form of Equation (3), where $f$ is differentiable in the interior and uniformly Hölder continuous up to the boundary.

In Theorems 1.2 and 1.4, the high-order vanishing condition (4) allows us to obtain uniqueness results without any extra assumption on $f$. This phenomenon is only found in autonomous equations like (3). For general ODEs of the form

$$
\frac{d^{n} u}{d t^{n}}=f\left(t, u, \frac{d u}{d t}, \ldots, \frac{d^{n-1} u}{d t^{n-1}}\right),
$$

such results cannot be expected because there is more than one expression for $f$. For example:

Example 3. Let $u(t)=t^{4}$. It satisfies the initial conditions

$$
u(0)=u^{\prime}(0)=u^{\prime \prime}(0)=u^{(3)}(0)=0 \quad \text { and } \quad u^{(4)}(0)=24 .
$$

Its derivatives are

$$
u^{\prime}(t)=4 t^{3} \quad \text { and } \quad u^{\prime \prime}(t)=12 t^{2} .
$$

We can express $u^{\prime \prime}$ as a function $f$ of $u$ and $u^{\prime}$ in different ways, such as

$$
u^{\prime \prime}(t)=\frac{192 u^{2}}{\left(u^{\prime}\right)^{2}}, \quad u^{\prime \prime}(t)=\frac{3\left(u^{\prime}\right)^{2}}{4 u}, \quad u^{\prime \prime}(t)=12\left(\frac{1}{4} u u^{\prime}\right)^{2 / 7},
$$


or

$$
u^{\prime \prime}(t)=12 u^{1 / 4}\left(\frac{1}{4} u^{\prime}\right)^{1 / 3}
$$

In the first two equations $f$ is not continuous at the origin, while in the last two equations $f$ is Hölder continuous at the origin. This simple example shows that the function $f$ can be expressed in various ways and that in order to study the uniqueness, we need to impose very specific assumptions on $f$.

Our work was motivated by that of Li and Nirenberg [2006], who studied a similar second-order PDE: $\Delta u=f(u)$, where $u=u(t, x) \in C^{\infty}\left(\mathbb{R}^{k+1}\right)$ has a nonvanishing partial derivative at 0 that can be expressed in the form $u(t, x)=a t^{m}+O\left(t^{m+1}\right)$, $a \neq 0, t \in \mathbb{R}$, and $x \in \mathbb{R}^{k}$. They showed that if two solutions $u$ and $v$ satisfy $u \geq v$, then $u \equiv v$. Theorem 1.2 can be viewed as an improvement of their result in the one-dimensional case to arbitrary order and without the comparison condition $u \geq v$.

\section{The proof of Lemma 1.3}

Without loss of generality, we can assume that $a>0$.

First, we show that $a$, the $m$-th derivative of $u$ at 0 , only depends on $m, n$, and the function $f$.

Define

$$
\tilde{x}=\left(\frac{u}{a}\right)^{1 / m} \text {. }
$$

Then

$$
\tilde{x}=\left(t^{m}+O\left(t^{m+1}\right)\right)^{1 / m}=t(1+O(t)) .
$$

This implies that

$$
\frac{\tilde{x}}{t} \rightarrow 1 \quad \text { as } \quad t \rightarrow 0
$$

We can also write $u=a \tilde{x}^{m}$. Taking the derivative with respect to $t$, we get

$$
\begin{aligned}
\frac{d u}{d t} & =a m \tilde{x}^{m-1} \frac{d \tilde{x}}{d t}, \\
a m t^{m-1}+O\left(t^{m}\right) & =a m \tilde{x}^{m-1} \frac{d \tilde{x}}{d t}, \\
\frac{t^{m-1}}{\tilde{x}^{m-1}}+O\left(\frac{t^{m}}{\tilde{x}^{m-1}}\right) & =\frac{d \tilde{x}}{d t} .
\end{aligned}
$$

In the second equation above and in the analysis that follows, we formally differentiate the Taylor expansion with the big- $O$ notation. A detailed discussion of this differentiation is provided in the Appendix. 
In light of (8), it follows that

$$
\left.\frac{d \tilde{x}}{d t}\right|_{t=0}=1
$$

By the inverse function theorem, $t$ can be expressed as a function of $\tilde{x}$ :

$$
t=\tilde{x}+O\left(\tilde{x}^{2}\right) .
$$

Then

$$
t^{m-n}=\left(\tilde{x}+O\left(\tilde{x}^{2}\right)\right)^{m-n}=\tilde{x}^{m-n}(1+O(\tilde{x})) .
$$

Similarly, $t^{m-n+1}=\tilde{x}^{m-n+1}(1+O(\tilde{x}))$. Thus

$$
\begin{aligned}
f(u) & =u^{(n)} \\
& =a m(m-1) \ldots(m-n+1) t^{m-n}+O\left(t^{m-n+1}\right) \\
& =a m(m-1) \ldots(m-n+1) \tilde{x}^{m-n}+O\left(\tilde{x}^{m-n+1}\right) \\
& =a m(m-1) \ldots(m-n+1)\left(\frac{u}{a}\right)^{(m-n) / m}+O\left(u^{(m-n+1) / m}\right) \\
& =a^{n / m} m(m-1) \ldots(m-n+1) u^{(m-n) / m}+O\left(u^{(m-n+1) / m}\right) .
\end{aligned}
$$

Therefore,

$$
a^{n / m}=\lim _{u \rightarrow 0} \frac{f(u)}{m(m-1) \ldots(m-n+1) u^{(m-n) / m}} .
$$

This shows that $a$ is completely determined by $m, n$, and the behavior of the function $f$ near 0 .

Next, we show that the $(m+1)$-th derivative of $u$ at 0 also only depends on $m, n$, and $f$.

Write $u$ as

$$
u(t)=a t^{m}+a_{m+1} t^{m+1}+O\left(t^{m+2}\right) .
$$

We will show that $a_{m+1}$ only depends on $m, n$, and the behavior of $f$ at 0 .

Express $t$ as

$$
t=\tilde{x}+b_{2} \tilde{x}^{2}+O\left(\tilde{x}^{3}\right)
$$

We would like to obtain an expression for $b_{2}$ in terms of the derivatives of $u$ at 0 . To do this, we take the derivative with respect to $t$ on both sides of

$$
\frac{d \tilde{x}}{d t} \cdot \frac{d t}{d \tilde{x}}=1
$$


By the product rule and chain rule, we have

$$
\begin{aligned}
\frac{d}{d t}\left(\frac{d \tilde{x}}{d t}\right) \cdot \frac{d t}{d \tilde{x}}+\frac{d \tilde{x}}{d t} \cdot \frac{d}{d t}\left(\frac{d t}{d \tilde{x}}\right) & =0, \\
\frac{d^{2} \tilde{x}}{d t^{2}} \cdot \frac{d t}{d \tilde{x}}+\frac{d \tilde{x}}{d t} \cdot\left(\frac{d^{2} t}{d \tilde{x}^{2}} \cdot \frac{d \tilde{x}}{d t}\right) & =0, \\
\frac{d^{2} \tilde{x}}{d t^{2}} \cdot \frac{d t}{d \tilde{x}}+\left(\frac{d \tilde{x}}{d t}\right)^{2} \cdot \frac{d^{2} t}{d \tilde{x}^{2}} & =0 .
\end{aligned}
$$

We would like to evaluate (14) at $t=0$.

From

$$
\begin{aligned}
\tilde{x} & =\left(\frac{u}{a}\right)^{1 / m}=\left(t^{m}+\frac{a_{m+1}}{a} t^{m+1}+O\left(t^{m+2}\right)\right)^{1 / m} \\
& =t\left(1+\frac{a_{m+1}}{a} t+O\left(t^{2}\right)\right)^{1 / m} \\
& =t\left(1+\frac{1}{m}\left(\frac{a_{m+1}}{a} t+O\left(t^{2}\right)\right)+\frac{1}{2} \cdot \frac{1}{m}\left(\frac{1}{m}-1\right)\left(\frac{a_{m+1}}{a} t+O\left(t^{2}\right)\right)^{2}+O\left(t^{3}\right)\right) \\
& =t+\frac{a_{m+1}}{m a} t^{2}+O\left(t^{3}\right),
\end{aligned}
$$

we know that

$$
\left.\frac{d^{2} \tilde{x}}{d t^{2}}\right|_{t=0}=\frac{2 a_{m+1}}{m a} .
$$

From (13) we know that

$$
\left.\frac{d t}{d \tilde{x}}\right|_{t=0}=1 \quad \text { and }\left.\quad \frac{d^{2} t}{d \tilde{x}^{2}}\right|_{t=0}=2 b_{2} .
$$

Thus if we evaluate (14) at $t=0$, we get

$$
\frac{2 a_{m+1}}{m a} \cdot 1+1 \cdot 2 b_{2}=0,
$$

and therefore

$$
b_{2}=-\frac{a_{m+1}}{m a} .
$$

Now, from (13) we have

$$
\begin{aligned}
t^{m-n} & =\left(\tilde{x}+b_{2} \tilde{x}^{2}+O\left(\tilde{x}^{3}\right)\right)^{m-n} \\
& =\tilde{x}^{m-n}\left[1+b_{2} \tilde{x}+O\left(\tilde{x}^{2}\right)\right]^{m-n} \\
& =\tilde{x}^{m-n}\left[1+(m-n)\left(b_{2} \tilde{x}+O\left(\tilde{x}^{2}\right)\right)+O\left(\tilde{x}^{2}\right)\right] \\
& =\tilde{x}^{m-n}+(m-n) b_{2} \tilde{x}^{m-n+1}+O\left(\tilde{x}^{m-n+2}\right) .
\end{aligned}
$$


Similarly,

$$
\begin{aligned}
& t^{m-n+1}=\tilde{x}^{m-n+1}+(m-n+1) b_{2} \tilde{x}^{m-n+2}+O\left(\tilde{x}^{m-n+3}\right), \\
& t^{m-n+2}=O\left(\tilde{x}^{m-n+2}\right) .
\end{aligned}
$$

Then from (12) and the above expressions for the powers of $t$, we have

$$
\begin{aligned}
u^{(n)}=a & (m-1) \ldots(m-n+1) t^{m-n} \\
& +a_{m+1}(m+1) m \ldots(m-n+2) t^{m-n+1}+O\left(t^{m-n+2}\right) \\
=a & (m-1) \ldots(m-n+1)\left(\tilde{x}^{m-n}+(m-n) b_{2} \tilde{x}^{m-n+1}+O\left(\tilde{x}^{m-n+2}\right)\right) \\
& +a_{m+1}(m+1) m \ldots(m-n+2) \\
& \quad \times\left(\tilde{x}^{m-n+1}+(m-n+1) b_{2} \tilde{x}^{m-n+2}+O\left(\tilde{x}^{m-n+3}\right)\right) \\
& +O\left(\tilde{x}^{m-n+2}\right) \\
=a & (m-1) \ldots(m-n+1) \tilde{x}^{m-n} \\
& +\left(a m(m-1) \ldots(m-n) b_{2}+a_{m+1}(m+1) m \ldots(m-n+2)\right) \tilde{x}^{m-n+1} \\
& +O\left(\tilde{x}^{m-n+2}\right) .
\end{aligned}
$$

Thus, in view of $f(u)=u^{(n)}$ and (7), we have

$$
\begin{aligned}
f(u)= & a m(m-1) \ldots(m-n+1) \tilde{x}^{m-n} \\
& +\left(a m(m-1) \ldots(m-n) b_{2}+a_{m+1}(m+1) m \ldots(m-n+2)\right) \tilde{x}^{m-n+1} \\
& +O\left(\tilde{x}^{m-n+2}\right) \\
= & a m(m-1) \ldots(m-n+1)\left(\frac{u}{a}\right)^{\frac{m-n}{m}} \\
& +\left(a m(m-1) \ldots(m-n) b_{2}+a_{m+1}(m+1) m \ldots(m-n+2)\right)\left(\frac{u}{a}\right)^{\frac{m-n+1}{m}} \\
& +O\left(\left(\frac{u}{a}\right)^{\frac{m-n+2}{m}}\right) \\
= & a^{\frac{n}{m}} m(m-1) \ldots(m-n+1) u^{\frac{m-n}{m}} \\
& +\left(a^{\frac{n-1}{m}} m(m-1) \ldots(m-n) b_{2}+a^{\frac{n-m-1}{m}} a_{m+1}(m+1) m \ldots(m-n+2)\right) \\
& +O\left(u^{\frac{m-n+2}{m}}\right) .
\end{aligned}
$$

This means that

$$
\begin{aligned}
a^{\frac{n-1}{m}} m(m-1) \ldots(m-n) b_{2} & +a^{\frac{n-m-1}{m}} a_{m+1}(m+1) m \ldots(m-n+2) \\
& =\lim _{u \rightarrow 0} \frac{f(u)-a^{n / m} m(m-1) \ldots(m-n+1) u^{\frac{m-n}{m}}}{u^{\frac{m-n+1}{m}}} .
\end{aligned}
$$


By (16), this can be written as

$$
\begin{gathered}
a^{\frac{n-1}{m}} m(m-1) \ldots(m-n)\left(-\frac{a_{m+1}}{m a}\right)+a^{\frac{n-m-1}{m}} a_{m+1}(m+1) m \ldots(m-n+2) \\
=\lim _{u \rightarrow 0} \frac{f(u)-a^{\frac{n}{m}} m(m-1) \ldots(m-n+1) u^{\frac{m-n}{m}}}{u^{\frac{m-n+1}{m}}} .
\end{gathered}
$$

After collecting similar terms, we get

$$
\begin{aligned}
a_{m+1} a^{\frac{n-m-1}{m}}((m+1) m \ldots(m-n+2)-(m-1)(m-2) \ldots(m-n)) \\
=\lim _{u \rightarrow 0} \frac{f(u)-a^{\frac{n}{m}} m(m-1) \ldots(m-n+1) u^{\frac{m-n}{m}}}{u^{\frac{m-n+1}{m}}} .
\end{aligned}
$$

Consequently,

$$
a_{m+1}=\frac{a^{\frac{m-n+1}{m}}\left(\lim _{u \rightarrow 0} \frac{f(u)-a^{\frac{n}{m}} m(m-1) \ldots(m-n+1) u^{\frac{m-n}{m}}}{u^{\frac{m-n+1}{m}}}\right)}{(m+1) m \ldots(m-n+2)-(m-1)(m-2) \ldots(m-n)} .
$$

Since we have proved that $a$ only depends on $m, n$, and $f$, Equation (17) shows that $a_{m+1}$ is also completely determined by $m, n$, and the behavior of $f$ near 0 . By (16), this also shows that $b_{2}$ depends only on $m, n$, and $f$.

Now we will use mathematical induction to show that all the derivatives of $u$ at 0 of order higher than $m$ are completely determined by $m, n$, and $f$.

Express $u$ and $t$ as

$$
u(t)=a t^{m}+a_{m+1} t^{m+1}+\cdots+a_{m+k} t^{m+k}+a_{m+k+1} t^{m+k+1}+O\left(t^{m+k+2}\right)
$$

and

$$
t=\tilde{x}+b_{2} \tilde{x}^{2}+\cdots+b_{k+1} \tilde{x}^{k+1}+b_{k+2} \tilde{x}^{k+2}+O\left(\tilde{x}^{k+3}\right) .
$$

Suppose that for $k \geq 1, a, a_{m+1}, \ldots, a_{m+k}, b_{2}, \ldots, b_{k+1}$ are all determined only by $m, n$, and $f$; we will show that $a_{m+k+1}$ and $b_{k+2}$ also are determined only by $m, n$, and $f$.

We start by obtaining an expression for $b_{k+2}$ in terms of $a_{m+1}, \ldots, a_{m+k}$ and $a_{m+k+1}$.

Taking the derivative with respect to $t$ on both sides of (14), we obtain

$$
0=\frac{d^{3} \tilde{x}}{d t^{3}} \cdot \frac{d t}{d \tilde{x}}+\frac{d^{2} \tilde{x}}{d t^{2}} \cdot\left(\frac{d^{2} t}{d \tilde{x}^{2}} \cdot \frac{d \tilde{x}}{d t}\right)+2 \cdot \frac{d \tilde{x}}{d t} \cdot \frac{d^{2} \tilde{x}}{d t^{2}} \cdot \frac{d^{2} t}{d \tilde{x}^{2}}+\left(\frac{d \tilde{x}}{d t}\right)^{2} \cdot\left(\frac{d^{3} t}{d \tilde{x}^{3}} \cdot \frac{d \tilde{x}}{d t}\right)
$$


and

$$
0=\frac{d^{3} \tilde{x}}{d t^{3}} \cdot \frac{d t}{d \tilde{x}}+3 \cdot \frac{d \tilde{x}}{d t} \cdot \frac{d^{2} \tilde{x}}{d t^{2}} \cdot \frac{d^{2} t}{d \tilde{x}^{2}}+\left(\frac{d \tilde{x}}{d t}\right)^{3} \cdot \frac{d^{3} t}{d \tilde{x}^{3}}
$$

Taking the derivative of both sides of these equations, we get

$$
\begin{aligned}
0=( & \left.\frac{d^{4} \tilde{x}}{d t^{4}} \cdot \frac{d t}{d \tilde{x}}+\frac{d^{3} \tilde{x}}{d t^{3}} \cdot \frac{d^{2} t}{d \tilde{x}^{2}} \cdot \frac{d \tilde{x}}{d t}\right) \\
& +3\left(\frac{d^{2} \tilde{x}}{d t^{2}} \cdot \frac{d^{2} \tilde{x}}{d t^{2}} \cdot \frac{d^{2} t}{d \tilde{x}^{2}}+\frac{d \tilde{x}}{d t} \cdot \frac{d^{3} \tilde{x}}{d t^{3}} \cdot \frac{d^{2} t}{d \tilde{x}^{2}}+\frac{d \tilde{x}}{d t} \cdot \frac{d^{2} \tilde{x}}{d t^{2}} \cdot \frac{d^{3} t}{d \tilde{x}^{3}} \cdot \frac{d \tilde{x}}{d t}\right) \\
& +\left(3\left(\frac{d \tilde{x}}{d t}\right)^{2} \cdot \frac{d^{2} \tilde{x}}{d t^{2}} \cdot \frac{d^{3} t}{d \tilde{x}^{3}}+\left(\frac{d \tilde{x}}{d t}\right)^{3} \cdot \frac{d^{4} t}{d \tilde{x}^{4}} \cdot \frac{d \tilde{x}}{d t}\right), \\
0= & \frac{d^{4} \tilde{x}}{d t^{4}} \cdot \frac{d t}{d \tilde{x}}+4 \cdot \frac{d^{3} \tilde{x}}{d t^{3}} \cdot \frac{d \tilde{x}}{d t} \cdot \frac{d^{2} t}{d \tilde{x}^{2}}+3 \cdot \frac{d^{2} \tilde{x}}{d t^{2}} \cdot \frac{d^{2} \tilde{x}}{d t^{2}} \cdot \frac{d^{2} t}{d \tilde{x}^{2}} \\
& +6\left(\frac{d \tilde{x}}{d t}\right)^{2} \cdot \frac{d^{2} \tilde{x}}{d t^{2}} \cdot \frac{d^{3} t}{d \tilde{x}^{3}}+\left(\frac{d \tilde{x}}{d t}\right)^{4} \cdot \frac{d^{4} t}{d \tilde{x}^{4}} .
\end{aligned}
$$

If we take the derivative $k$ times with respect to $t$ and collect the similar terms after each differentiation as shown above, eventually we arrive at an expression of the form

$$
\begin{aligned}
0=\frac{d^{k+2} \tilde{x}}{d t^{k+2}} \cdot \frac{d t}{d \tilde{x}} & \\
& +\left(\text { terms involving } \frac{d^{k+1} \tilde{x}}{d t^{k+1}}, \frac{d^{k} \tilde{x}}{d t^{k}}, \ldots, \frac{d \tilde{x}}{d t}, \frac{d t}{d \tilde{x}}, \frac{d^{2} t}{d \tilde{x}^{2}}, \ldots, \frac{d^{k+1} t}{d \tilde{x}^{k+1}}\right) \\
& +\left(\frac{d \tilde{x}}{d t}\right)^{k+2} \cdot \frac{d^{k+2} t}{d \tilde{x}^{k+2}}
\end{aligned}
$$

From (19) we know that

$$
\begin{aligned}
\left.\frac{d t}{d \tilde{x}}\right|_{t=0} & =1, \\
\left.\frac{d^{2} t}{d \tilde{x}^{2}}\right|_{t=0} & =2 b_{2},
\end{aligned}
$$

$$
\begin{aligned}
& \left.\frac{d^{k+1} t}{d \tilde{x}^{k+1}}\right|_{t=0}=(k+1) ! b_{k+1}, \\
& \left.\frac{d^{k+2} t}{d \tilde{x}^{k+2}}\right|_{t=0}=(k+2) ! b_{k+2}
\end{aligned}
$$


Then we look at $\left.\frac{d \tilde{x}}{d t}\right|_{t=0}, \ldots,\left.\frac{d^{k+1} \tilde{x}}{d t^{k+1}}\right|_{t=0}$, and $\left.\frac{d^{k+2} \tilde{x}}{d t^{k+2}}\right|_{t=0}$. By (7) and (18),

$$
\begin{aligned}
\tilde{x}=( & \left.\frac{a t^{m}+a_{m+1} t^{m+1}+\cdots+a_{m+k} t^{m+k}+a_{m+k+1} t^{m+k+1}+O\left(t^{m+k+2}\right)}{a}\right)^{1 / m} \\
=t & \left(1+\frac{a_{m+1}}{a} t+\frac{a_{m+2}}{a} t^{2}+\cdots+\frac{a_{m+k}}{a} t^{k}+\frac{a_{m+k+1}}{a} t^{k+1}+O\left(t^{k+2}\right)\right)^{1 / m} \\
=t & \left\{1+\frac{1}{m}\left[\frac{a_{m+1}}{a} t+\frac{a_{m+2}}{a} t^{2}+\cdots+\frac{a_{m+k}}{a} t^{k}+\frac{a_{m+k+1}}{a} t^{k+1}+O\left(t^{k+2}\right)\right]\right. \\
& +\frac{1}{2} \cdot \frac{1}{m}\left(\frac{1}{m}-1\right) \\
& \left.+\cdots \frac{a_{m+1}}{a} t+\frac{a_{m+2}}{a} t^{2}+\cdots+\frac{a_{m+k}}{a} t^{k}+\frac{a_{m+k+1}}{a} t^{k+1}+O\left(t^{k+2}\right)\right]^{2} \\
& +\frac{1}{(k+1) !} \cdot \frac{1}{m} \cdot\left(\frac{1}{m}-1\right) \cdots\left(\frac{1}{m}-k\right) \\
& \quad\left[\frac{a_{m+1}}{a} t+\frac{a_{m+2}}{a} t^{2}+\cdots+\frac{a_{m+k}}{a} t^{k}+\frac{a_{m+k+1}}{a} t^{k+1}+O\left(t^{k+2}\right)\right]^{k+1} \\
& \left.O\left(t^{k+2}\right)\right\}
\end{aligned}
$$

After collecting similar terms, we can write

$$
\tilde{x}=t+\lambda_{2} t^{2}+\lambda_{3} t^{3}+\cdots+\lambda_{k+1} t^{k+1}+\left(\frac{a_{m+k+1}}{m a}+\lambda_{k+2}\right) t^{k+2}+O\left(t^{k+3}\right)
$$

where

- $\lambda_{2}$ is a constant involving $m, a$, and $a_{m+1}$,

- $\lambda_{3}$ is a constant involving $m, a, a_{m+1}$ and $a_{m+2}$,

- $\lambda_{k+1}$ is a constant involving $m, a, a_{m+1}, \ldots, a_{m+k-1}, a_{m+k}$, and

- $\lambda_{k+2}$ is a constant involving $m, a, a_{m+1}, \ldots, a_{m+k-1}, a_{m+k}$.

By the inductive hypothesis, $\lambda_{2}, \lambda_{3}, \ldots, \lambda_{k+1}, \lambda_{k+2}$ are all constants that only depend on $m, n$, and the function $f$. 
From (23), we obtain

$$
\begin{gathered}
\left.\frac{d \tilde{x}}{d t}\right|_{t=0}=1, \\
\left.\frac{d^{2} \tilde{x}}{d t^{2}}\right|_{t=0}=2 \lambda_{2},
\end{gathered}
$$

(24)

$$
\begin{aligned}
& \left.\frac{d^{k+1} \tilde{x}}{d t^{k+1}}\right|_{t=0}=(k+1) ! \lambda_{k+1}, \\
& \left.\frac{d^{k+2} \tilde{x}}{d t^{k+2}}\right|_{t=0}=(k+2) !\left(\frac{a_{m+k+1}}{m a}+\lambda_{k+2}\right) .
\end{aligned}
$$

Now we evaluate (21) at $t=0$ and make use of (22) and (24):

$$
\begin{aligned}
0=(k+2) !( & \left.\frac{a_{m+k+1}}{m a}+\lambda_{k+2}\right) \cdot 1 \\
& +\left(\text { terms involving } b_{2}, \ldots, b_{k+1}, \lambda_{2}, \ldots, \lambda_{k+1}\right)+1 \cdot(k+2) ! b_{k+2} .
\end{aligned}
$$

Thus we obtain

$$
b_{k+2}=-\frac{a_{m+k+1}}{m a}+Q,
$$

where $Q$ is a constant depending on $b_{2}, \ldots, b_{k+1}, \lambda_{2}, \ldots, \lambda_{k+1}, \lambda_{k+2}$, and hence $Q$ is completely determined by $m, n$, and $f$.

Next we will analyze $a_{m+k+1}$. From (19) we have

$$
\begin{aligned}
t^{m-n}= & \tilde{x}^{m-n}\left(1+b_{2} \tilde{x}+\cdots+b_{k+1} \tilde{x}^{k}+b_{k+2} \tilde{x}^{k+1}+O\left(\tilde{x}^{k+2}\right)\right)^{m-n} \\
=\tilde{x}^{m-n}\left\{1+(m-n)\left(b_{2} \tilde{x}+\cdots+b_{k+1} \tilde{x}^{k}+b_{k+2} \tilde{x}^{k+1}+O\left(\tilde{x}^{k+2}\right)\right)\right. & \\
& +\frac{(m-n)(m-n-1)}{2}\left(b_{2} \tilde{x}+\cdots+b_{k+1} \tilde{x}^{k}+b_{k+2} \tilde{x}^{k+1}+O\left(\tilde{x}^{k+2}\right)\right)^{2}+\cdots \\
& +\frac{(m-n)(m-n-1) \ldots(m-n-k)}{(k+1) !} \\
& \quad\left(b_{2} \tilde{x}+\cdots+b_{k+1} \tilde{x}^{k}+b_{k+2} \tilde{x}^{k+1}+O\left(\tilde{x}^{k+2}\right)\right)^{k+1} \\
& \left.+O\left(\tilde{x}^{k+2}\right)\right\} .
\end{aligned}
$$

After collecting similar terms, we can express $t^{m-n}$ as

$$
\begin{aligned}
t^{m-n}=\tilde{x}^{m-n}\left\{1+c_{1, m-n} \tilde{x}+c_{2, m-n}\right. & \tilde{x}^{2}+\cdots+c_{k, m-n} \tilde{x}^{k} \\
& \left.+\left((m-n) b_{k+2}+c_{k+1, m-n}\right) \tilde{x}^{k+1}+O\left(\tilde{x}^{k+2}\right)\right\},
\end{aligned}
$$


where $c_{1, m-n}$ is a constant depending on $m$ and $b_{2} ; c_{2, m-n}$ is a constant depending on $m, b_{2}$, and $b_{3} ; \ldots ; c_{k, m-n}$ is a constant depending on $m, b_{2}, \ldots, b_{k+1} ; c_{k+1, m-n}$ is a constant depending on $m, b_{2}, \ldots, b_{k+1}$.

By the inductive hypothesis, $c_{1, m-n}, c_{2, m-n}, \ldots, c_{k, m-n}$ and $c_{k+1, m-n}$ are all determined only by $m, n$, and $f$. Thus we have

$$
\begin{aligned}
t^{m-n}=\tilde{x}^{m-n}+c_{1, m-n} \tilde{x}^{m-n+1}+c_{2, m-n} \tilde{x}^{m-n+2}+\cdots+c_{k, m-n} \tilde{x}^{m-n+k} \\
+\left((m-n) b_{k+2}+c_{k+1, m-n}\right) \tilde{x}^{m-n+k+1}+O\left(\tilde{x}^{m-n+k+2}\right),
\end{aligned}
$$

where $c_{1, m-n}, c_{2, m-n}, \ldots, c_{k, m-n}$ and $c_{k+1, m-n}$ are constants depending on $m, n$, and $f$.

By the same type of analysis we obtain similar expressions for the other powers of $t$ :

$$
\begin{array}{r}
t^{m-n+1}=\tilde{x}^{m-n+1}+c_{1, m-n+1} \tilde{x}^{m-n+2}+c_{2, m-n+1} \tilde{x}^{m-n+3}+\cdots \\
+c_{k, m-n+1} \tilde{x}^{m-n+k+1}+\left((m-n+1) b_{k+2}+c_{k+1, m-n+1}\right) \tilde{x}^{m-n+k+2} \\
+O\left(\tilde{x}^{m-n+k+3}\right)
\end{array}
$$

where $c_{1, m-n+1}, c_{2, m-n+1}, \ldots, c_{k, m-n+1}$, and $c_{k+1, m-n+1}$ are constants depending on $m, n$, and $f$.

$$
\begin{array}{r}
t^{m-n+2}=\tilde{x}^{m-n+2}+c_{1, m-n+2} \tilde{x}^{m-n+3}+c_{2, m-n+2} \tilde{x}^{m-n+4}+\cdots \\
+c_{k, m-n+2} \tilde{x}^{m-n+k+2}+\left((m-n+2) b_{k+2}+c_{k+1, m-n+2}\right) \tilde{x}^{m-n+k+3} \\
+O\left(\tilde{x}^{m-n+k+4}\right)
\end{array}
$$

where $c_{1, m-n+2}, c_{2, m-n+2}, \ldots, c_{k, m-n+2}$ and $c_{k+1, m-n+2}$ are constants depending on $m, n$, and $f$. Proceeding inductively,

$$
\begin{array}{r}
t^{m-n+k}=\tilde{x}^{m-n+k}+c_{1, m-n+k} \tilde{x}^{m-n+k+1}+c_{2, m-n+k} \tilde{x}^{m-n+k+2}+\cdots \\
+c_{k, m-n+k} \tilde{x}^{m-n+2 k}+\left((m-n+k) b_{k+2}+c_{k+1, m-n+k}\right) \tilde{x}^{m-n+2 k+1} \\
+O\left(\tilde{x}^{m-n+2 k+2}\right)
\end{array}
$$

where $c_{1, m-n+k}, c_{2, m-n+k}, \ldots, c_{k, m-n+k}$ and $c_{k+1, m-n+k}$ are constants depending on $m, n$, and $f$.

$$
\begin{aligned}
& t^{m-n+k+1}=\tilde{x}^{m-n+k+1}+c_{1, m-n+k+1} \tilde{x}^{m-n+k+2} \\
& \quad+c_{2, m-n+k+1} \tilde{x}^{m-n+k+3}+\cdots+c_{k, m-n+k+1} \tilde{x}^{m-n+2 k+1} \\
& +\left((m-n+k+1) b_{k+2}+c_{k+1, m-n+k+1}\right) \tilde{x}^{m-n+2 k+2}+O\left(\tilde{x}^{m-n+2 k+3}\right)
\end{aligned}
$$

where $c_{1, m-n+k+1}, \ldots, c_{k, m-n+k+1}$ and $c_{k+1, m-n+k+1}$ are constants depending on $m, n$, and $f$.

$$
t^{m-n+k+2}=O\left(\tilde{x}^{m-n+k+2}\right) .
$$


From (18) we obtain

$$
\begin{aligned}
u^{(n)}= & a m(m-1) \ldots(m-n+1) t^{m-n}+a_{m+1}(m+1) m \ldots(m-n+2) t^{m-n+1} \\
& +\cdots+a_{m+k}(m+k)(m+k-1) \ldots(m-n+k+1) t^{m-n+k} \\
& +a_{m+k+1}(m+k+1)(m+k) \ldots(m-n+k+2) t^{m-n+k+1}+O\left(t^{m-n+k+2}\right) .
\end{aligned}
$$

Then by (26) to (31), we can write

$$
\begin{aligned}
u^{(n)}= & a m(m-1) \ldots(m-n+1)\left\{\tilde{x}^{m-n}+c_{1, m-n} \tilde{x}^{m-n+1}+c_{2, m-n} \tilde{x}^{m-n+2}+\cdots\right. \\
& \left.+c_{k, m-n} \tilde{x}^{m-n+k}+\left((m-n) b_{k+2}+c_{k+1, m-n}\right) \tilde{x}^{m-n+k+1}+O\left(\tilde{x}^{m-n+k+2}\right)\right\} \\
& +a_{m+1}(m+1) m \ldots(m-n+2)\left\{\tilde{x}^{m-n+1}+c_{1, m-n+1} \tilde{x}^{m-n+2}\right. \\
& +c_{2, m-n+1} \tilde{x}^{m-n+3}+\ldots+c_{k, m-n+1} \tilde{x}^{m-n+k+1} \\
& \left.+\left((m-n+1) b_{k+2}+c_{k+1, m-n+1}\right) \tilde{x}^{m-n+k+2}+O\left(\tilde{x}^{m-n+k+3}\right)\right\}+\cdots \\
& +a_{m+k}(m+k)(m+k-1) \ldots(m+k-n+1)\left\{\tilde{x}^{m-n+k}\right. \\
& +c_{1, m-n+k} \tilde{x}^{m-n+k+1}+c_{2, m-n+k} \tilde{x}^{m-n+k+2}+\ldots \\
& +c_{k, m-n+k} \tilde{x}^{m-n+2 k}+\left((m-n+k) b_{k+2}+c_{k+1, m-n+k}\right) \tilde{x}^{m-n+2 k+1} \\
& \left.+O\left(\tilde{x}^{m-n+2 k+2}\right)\right\} \\
& +a_{m+k+1}(m+k+1)(m+k) \ldots(m+k-n+2)\left\{\tilde{x}^{m-n+k+1}\right. \\
& +c_{1, m-n+k+1} \tilde{x}^{m-n+k+2}+c_{2, m-n+k+1} \tilde{x}^{m-n+k+3}+\cdots \\
& +c_{k, m-n+k+1} \tilde{x}^{m-n+2 k+1} \\
& \left.+\left((m-n+k+1) b_{k+2}+c_{k+1, m-n+k+1}\right) \tilde{x}^{m-n+2 k+2}+O\left(\tilde{x}^{m-n+2 k+3}\right)\right\} \\
& +O\left(\tilde{x}^{m-n+k+2}\right) \\
= & a m(m-1) \cdots(m-n+1) \tilde{x}^{m-n}+C\left(m, a, a_{m+1}, c_{1, m-n}\right) \tilde{x}^{m-n+1} \\
& +C\left(m, a, a_{m+1}, a_{m+2}, c_{1, m-n+1}, c_{2, m-n}\right) \tilde{x}^{m-n+2}+\cdots \\
& +C\left(m, a, a_{m+1}, \ldots, a_{m+k}, c_{k, m-n}, c_{k-1, m-n+1}, \ldots, c_{1, m-n+k-1}\right) \tilde{x}^{m-n+k} \\
& +\left(a m(m-1) \ldots(m-n) b_{k+2}\right. \\
& +a_{m+k+1}(m+k+1)(m+k) \ldots(m+k-n+2) \\
& \left.+C\left(m, a, a_{m+1}, \ldots, a_{m+k}, c_{k+1, m-n}, c_{k, m-n+1}, \ldots, c_{1, m-n+k}\right)\right) \tilde{x}^{m-n+k+1} \\
& O\left(\tilde{x}^{m-n+k+2}\right) . \\
& \\
&
\end{aligned}
$$


Here $C\left(m, a, a_{m+1}, c_{1, m-n}\right)$ is a constant depending on $m, a, a_{m+1}$, and $c_{1, m-n}$; we denote it as $p_{m-n+1}$ to simplify notations. Since $a, a_{m+1}$, and $c_{1, m-n}$ only depend on $m, n$, and $f$, we know that $p_{m-n+1}$ only depends on $m, n$, and $f$.

Similarly, the other constants $C\left(m, a, a_{m+1}, a_{m+2}, c_{1, m-n+1}, c_{2, m-n}\right), \ldots$, and $C\left(m, a, a_{m+1}, \ldots, a_{m+k}, c_{k+1, m-n}, c_{k, m-n+1}, \ldots, c_{1, m-n+k}\right)$ all depend on $m, n$, and $f$ only, and can be denoted simply as $p_{m-n+2}, \ldots, p_{m-n+k}$, and $p_{m-n+k+1}$. Thus we can rewrite the above equation as

$$
\begin{aligned}
u^{(n)}= & a m(m-1) \ldots(m-n+1) \tilde{x}^{m-n}+p_{m-n+1} \tilde{x}^{m-n+1} \\
& +p_{m-n+2} \tilde{x}^{m-n+2}+\cdots+p_{m-n+k} \tilde{x}^{m-n+k} \\
& +\left(a m(m-1) \ldots(m-n) b_{k+2}\right. \\
& \left.+a_{m+k+1}(m+k+1)(m+k) \ldots(m+k-n+2)+p_{m-n+k+1}\right) \tilde{x}^{m-n+k+1} \\
& +O\left(\tilde{x}^{m-n+k+2}\right) .
\end{aligned}
$$

Now because of $u^{(n)}=f(u)$ and definition (7), we have

$$
\begin{aligned}
f(u)= & a m(m-1) \ldots(m-n+1)\left(\frac{u}{a}\right)^{(m-n / m)}+p_{m-n+1}\left(\frac{u}{a}\right)^{(m-n+1 / m)} \\
& +p_{m-n+2}\left(\frac{u}{a}\right)^{(m-n+2) / m}+\cdots+p_{m-n+k}\left(\frac{u}{a}\right)^{(m-n+k) / m} \\
& +\left(a m(m-1) \ldots(m-n) b_{k+2}\right. \\
& \quad+a_{m+k+1}(m+k+1)(m+k) \cdots(m+k-n+2) \\
& \left.+p_{m-n+k+1}\right)\left(\frac{u}{a}\right)^{(m-n+k+1) / m} \\
+ & O\left(u^{(m-n+k+2) / m}\right) .
\end{aligned}
$$

Due to (25), we can rewrite this equation as

$$
\begin{aligned}
f(u)= & a m(m-1) \ldots(m-n+1)\left(\frac{u}{a}\right)^{(m-n) / m}+p_{m-n+1}\left(\frac{u}{a}\right)^{(m-n+1) / m} \\
& +p_{m-n+2}\left(\frac{u}{a}\right)^{(m-n+2) / m}+\cdots+p_{m-n+k}\left(\frac{u}{a}\right)^{(m-n+k) / m} \\
+ & \{((m+k+1)(m+k) \ldots(m+k-n+2) \\
& \quad-(m-1)(m-2) \ldots(m-n)) a_{m+k+1} \\
& \left.\quad+a m(m-1) \ldots(m-n) Q+p_{m-n+k+1}\right\}\left(\frac{u}{a}\right)^{(m-n+k+1) / m} \\
& +O\left(u^{(m-n+k+2) / m}\right) .
\end{aligned}
$$


From (33) we get

$$
\begin{aligned}
& ((m+k+1)(m+k) \ldots(m+k-n+2)-(m-1)(m-2) \ldots(m-n)) a_{m+k+1} \\
& \quad+\operatorname{am}(m-1) \ldots(m-n) Q+p_{m-n+k+1} \\
& \quad=\lim _{u \rightarrow 0} \frac{f(u)-a m \ldots(m-n+1)\left(\frac{u}{a}\right)^{\frac{m-n}{m}}-p_{m-n+1}\left(\frac{u}{a}\right)^{\frac{m-n+1}{m}}-\ldots-p_{m-n+k}\left(\frac{u}{a}\right)^{\frac{m-n+k}{m}}}{\left(\frac{u}{a}\right)^{\frac{m-n+k+1}{m}}} .
\end{aligned}
$$

Note that $(m+k+1)(m+k) \ldots(m+k-n+2)-(m-1)(m-2) \ldots(m-n) \neq 0$; then since the constants $Q, a, p_{m-n+1}, \ldots, p_{m-n+k+1}$ all depend only on $m, n$, and $f$, we know that $a_{m+k+1}$ only depends on $m, n$, and $f$. Consequently, $b_{k+2}$ also only depends on $m, n$, and $f$ because of (25).

Therefore, by mathematical induction, all derivatives of $f$ at 0 are determined completely by $m, n$, and $f$. This completes the proof of Lemma 1.3.

\section{The proofs of Theorems 1.2, 1.4 and 1.5}

Proof of Theorem 1.2. Suppose there are two solutions $u(t)$ and $v(t)$, both satisfying Equations (3) and (4). By Lemma 1.3, at $t=0, u$ and $v$ have the same derivative of any order. Let $w=u-v$; then

$$
w^{(k)}(0)=0 \quad \text { for any integer } \quad k \geq 0 .
$$

In order to apply Theorem 1.1, we need to show that $w$ satisfies Condition (2).

$$
w^{(n)}(t)=u^{(n)}(t)-v^{(n)}(t)=f(u(t))-f(v(t)) .
$$

Without loss of generality, we assume $a>0$. By Equation (10), we can write

$$
f(u)=\left[a^{n / m} m(m-1) \ldots(m-n+1) u^{(m-n) / m}+\alpha(u)\right]
$$

and

$$
f(v)=\left[a^{n / m} m(m-1) \ldots(m-n+1) v^{(m-n) / m}+\alpha(v)\right],
$$

where $\alpha$ is a function with the order

$$
\alpha(s)=O\left(s^{(m-n+1) / m}\right) .
$$

So we can write

$$
w^{(n)}(t)=a^{n / m} m(m-1) \ldots(m-n+1)\left(u^{(m-n) / m}-v^{(m-n) / m}\right)+(\alpha(u)-\alpha(v)) .
$$

If $m=n$, then

$$
a^{n / m} m(m-1) \ldots(m-n+1)\left(u^{(m-n) / m}-v^{(m-n) / m}\right)=0 .
$$


If $m>n$, by the mean value theorem,

$$
\left|u^{(m-n) / m}-v^{(m-n) / m}\right| \leq \frac{m-n}{m} \zeta^{-n / m}|u-v|,
$$

where $\zeta(t)$ is between $u(t)$ and $v(t)$. Since $u(t)=a t^{m}+O\left(t^{m+1}\right)$ and $v(t)=$ $a t^{m}+O\left(t^{m+1}\right)$, we know that $\zeta(t)=a t^{m}+O\left(t^{m+1}\right)$, which implies

$$
\zeta^{n / m}=a^{n / m} t^{n}(1+O(t)) \geq C t^{n}
$$

for some constant $C>0$ when $t$ is sufficiently small. Thus

$$
\zeta^{-n / m}|u-v| \leq C^{-1} \frac{|u-v|}{t^{n}}
$$

and by (36), we know that

$$
a^{n / m} m(m-1) \ldots(m-n+1)\left|u^{(m-n) / m}-v^{(m-n) / m}\right| \leq C \frac{|u-v|}{t^{n}}
$$

for another constant $C>0$.

Next, we estimate $|\alpha(u)-\alpha(v)|$.

From (3), we know that $f$ is differentiable with respect to $t$, since $u^{(n)}(t)$ is differentiable with respect to $t$. Condition (4) shows that $(d u / d t)(t) \neq 0$ when $t>0$ is sufficiently small. Then by the inverse function theorem, $t$ is differentiable with respect to $u$. Thus, when $u$ is small and positive, $f$ is differentiable with respect to $u$ and

$$
\frac{d f}{d u}=\frac{d f}{d t} \cdot \frac{d t}{d u}
$$

Then by (34), since $f$ is differentiable on a small interval $(0, \delta), \alpha$ is also differentiable on a small interval $(0, \delta)$. By the mean value theorem,

$$
\alpha(u)-\alpha(v)=\alpha^{\prime}(\eta)(u-v),
$$

where $\eta(t)$ is between $u(t)$ and $v(t)$. Because $u(t)=a t^{m}+O\left(t^{m+1}\right)$ and $v(t)=$ $a t^{m}+O\left(t^{m+1}\right)$, we know that $\eta(t)=a t^{m}+O\left(t^{m+1}\right) \geq C t^{m}$ when $t$ is small, and thus

$$
\eta^{-(n-1) / m}=O\left(t^{-(n-1)}\right) .
$$

From $\alpha(s)=O\left(s^{(m-n+1) / m}\right)$ we get $\alpha^{\prime}(s)=O\left(s^{-(n-1) / m}\right)$. Therefore

$$
\alpha^{\prime}(\eta)=O\left(\eta^{-(n-1) / m}\right)=O\left(t^{-(n-1)}\right) .
$$

Thus for some $C>0$,

$$
\begin{aligned}
|\alpha(u)-\alpha(v)| & \leq C t^{-(n-1)}|u-v| \\
& \leq C t^{-n}|u-v| \quad \text { since } \quad 0<t<1 .
\end{aligned}
$$


Combining Equations (35), (37), and (38), we conclude

$$
\left|w^{(n)}(t)\right| \leq C \frac{|u(t)-v(t)|}{t^{n}}=C \frac{|w(t)|}{t^{n}} .
$$

Finally, extend the domain of $w(t)$ to $[-1,1]$ by defining $w(t)=w(-t)$ when $-1 \leq t<0$. Then $w \in C^{\infty}([-1,1])$ and it satisfies Condition (2). By Theorem 1.1, $w \equiv 0$, which means $u \equiv v$.

This completes the proof of Theorem 1.2.

Proof of Theorem 1.4. Without loss of generality we assume $a>0$. We apply the same analysis as in the proof of Lemma 1.3 to $u_{1}$ and $u_{2}$, respectively. Similar to (11), we have

$$
\begin{aligned}
a^{n / m} & =\lim _{u_{1} \rightarrow 0} \frac{f\left(u_{1}\right)}{m(m-1) \ldots(m-n+1) u_{1}^{(m-n) / m}} \\
& =\lim _{s \rightarrow 0} \frac{f(s)}{m(m-1) \ldots(m-n+1) s^{(m-n) / m}}
\end{aligned}
$$

and

$$
b^{n / l}=\lim _{u_{2} \rightarrow 0} \frac{f\left(u_{2}\right)}{l(l-1) \ldots(l-n+1) u_{2}^{(l-n) / l}}=\lim _{s \rightarrow 0} \frac{f(s)}{l(l-1) \ldots(l-n+1) s^{(l-n) / l}} .
$$

Suppose $m \neq l$; without loss of generality we assume $m<l$. Dividing the two equations, we get

$$
\begin{aligned}
a^{n / m} b^{-n / l} & =\frac{l(l-1) \ldots(l-n+1)}{m(m-1) \ldots(m-n+1)} \lim _{s \rightarrow 0} s^{(l-n) / l-(m-n) / m} \\
& =\frac{l(l-1) \ldots(l-n+1)}{m(m-1) \ldots(m-n+1)} \lim _{s \rightarrow 0} s^{n / m-n / l} .
\end{aligned}
$$

Since $m<l, \lim _{s \rightarrow 0} s^{n / m-n / l}=0$. However, $a^{n / m} b^{-n / l} \neq 0$. This is a contradiction.

Therefore $m=l$, and consequently $a=b$. Then by Theorem 1.2, we know that $u_{1} \equiv u_{2}$ for small $t$.

Proof of Theorem 1.5. The proof of Lemma 1.3 shows that near $0, t$ is a function of $u$, and therefore $u^{(n)}(t)$ can be expressed as a function $f$ of $u$. Thus (3) holds when $t>0$ is small. From Condition (4), we define $f(0)=0$.

By the first two equations in (10) and the discussions in the Appendix, we know that there is a function $h$ that is $C^{1}$ on the closed interval $[0, \epsilon]$ for some $\epsilon>0$, such that

$$
f(u)=\operatorname{am}(m-1) \ldots(m-n+1) \tilde{x}^{m-n}+h(\tilde{x}) \tilde{x}^{m-n} .
$$

By definition (7), we have

$$
f(u)=a m(m-1) \ldots(m-n+1)\left(\frac{u}{a}\right)^{(m-n) / m}+h\left(\left(\frac{u}{a}\right)^{1 / m}\right)\left(\frac{u}{a}\right)^{(m-n) / m} .
$$


Since $0 \leq(m-n) / m<1$ and $0<1 / m<1$, it is well known that $u^{(m-n) / m}$ and $u^{1 / m}$ are Hölder continuous on the closed interval $[0,1]$ with Hölder coefficients $(m-n) / m$ and $1 / m$, respectively. This implies that the first term in (39) is Hölder continuous on $[0,1]$.

Since $h$ is $C^{1}$ on $[0, \epsilon]$, it is also Hölder continuous on $[0, \epsilon]$. Then since the composition of two Hölder continuous functions is Hölder continuous, we know that $h\left((u / a)^{1 / m}\right)$ is Hölder continuous with respect to $u$ on a closed interval $[0, \delta]$ with $\delta>0$. Next, because the product of two Hölder continuous functions is also Hölder continuous, we know that $h\left((u / a)^{1 / m}\right) \cdot(u / a)^{(m-n) / m}$ is Hölder continuous. Thus the second term in (39) is Hölder continuous on $[0, \delta]$.

Therefore, $f$ is Hölder continuous on $[0, \delta]$ and the theorem is proved.

\section{Appendix: Differentiation of the Taylor expansion}

We will discuss the regularity of the remainder term in the Taylor expansion of a function that is used in the proof of Theorem 1.5 and the differentiation of the Taylor expansion that is frequently used in the proof of Lemma 1.3.

In general, consider a function $g(x) \in C^{k+1}([a, b])$; by the Taylor theorem, we can write

$$
\begin{aligned}
& g(x)=g(a)+g^{\prime}(a)(x-a) \\
& \quad+\frac{g^{\prime \prime}(a)}{2 !}(x-a)^{2}+\cdots+\frac{g^{(k)}(a)}{k !}(x-a)^{k}+h(x)(x-a)^{k},
\end{aligned}
$$

where $\lim _{x \rightarrow a} h(x)=0$. An explicit expression for $h(x)$ is

$$
h(x)=\frac{g^{(k+1)}(\xi)}{(k+1) !}(x-a),
$$

where $a<\xi<x$.

From (40) we know that $h(x)$ is $C^{1}$ on $(a, b]$. Next we show that it is actually $C^{1}$ up to the boundary, on $[a, b]$.

Taking the derivative on both sides of (40), we get

$$
\begin{aligned}
g^{\prime}(x)=g^{\prime}(a)+ & g^{\prime \prime}(a)(x-a)+\cdots \\
& +\frac{g^{(k)}(a)}{(k-1) !}(x-a)^{k-1}+h^{\prime}(x)(x-a)^{k}+k h(x)(x-a)^{k-1} .
\end{aligned}
$$

Define $h(a)=0$, so $h$ is continuous on $[a, b]$. Write

$$
P(x)=g(a)+g^{\prime}(a)(x-a)+\frac{g^{\prime \prime}(a)}{2 !}(x-a)^{2}+\cdots+\frac{g^{(k)}(a)}{k !}(x-a)^{k} ;
$$

then $h(x)=\frac{g(x)-P(x)}{(x-a)^{k}}$. By the definition of limits, 
(43)

$$
\begin{aligned}
h^{\prime}(a) & =\lim _{x \rightarrow a} \frac{h(x)-h(a)}{x-a}=\lim _{x \rightarrow a} \frac{g(x)-P(x)}{(x-a)^{k+1}} \\
& \vdots \\
& =\frac{g^{(k+1)}(a)-P^{(k+1)}(a)}{(k+1) !} \text { applying l'Hospital's rule } k+1 \text { times } \\
& =\frac{g^{(k+1)}(a)}{(k+1) !}
\end{aligned}
$$

where we have used the fact that $P^{(k+1)}(a)=0$.

When $x>a$,

$$
\begin{aligned}
h^{\prime}(x) & =\frac{d}{d x}\left(\frac{g(x)-P(x)}{(x-a)^{k}}\right) \\
& =\frac{\left(g^{\prime}(x)-P^{\prime}(x)\right)(x-a)^{k}-(g(x)-P(x)) k(x-a)^{k-1}}{(x-a)^{2 k}} \\
& =\frac{g^{\prime}(x)-P^{\prime}(x)}{(x-a)^{k}}-\frac{k(g(x)-P(x))}{(x-a)^{k+1}} .
\end{aligned}
$$

By repeatedly applying l'Hospital's rule, we know that

$$
\lim _{x \rightarrow a} \frac{g^{\prime}(x)-P^{\prime}(x)}{(x-a)^{k}}=\frac{g^{(k+1)}(a)-P^{(k+1)}(a)}{k !}=\frac{g^{(k+1)}(a)}{k !}
$$

and

$$
\lim _{x \rightarrow a} \frac{k(g(x)-P(x))}{(x-a)^{k+1}}=\frac{k\left(g^{(k+1)}(a)-P^{(k+1)}(a)\right)}{(k+1) !}=\frac{k g^{(k+1)}(a)}{(k+1) !} .
$$

Therefore

$$
\lim _{x \rightarrow a} h^{\prime}(x)=\frac{g^{(k+1)}(a)}{k !}-\frac{k g^{(k+1)}(a)}{(k+1) !}=\frac{g^{(k+1)}(a)}{(k+1) !} .
$$

Equations (43) and (44) show that $h(x)$ is $C^{1}$ on the closed interval $[a, b]$.

Furthermore, we know that for any $x \in[a, b],\left|h^{\prime}(x)\right| \leq C$ for some constant $C_{1}$, and thus

$$
\left|h^{\prime}(x)(x-a)^{k}\right| \leq C_{1}|x-a|^{k} .
$$

Since $g(x) \in C^{k+1}([a, b])$, from (41) we know that $|h(x)| \leq C_{2}|x-a|$ for some constant $C_{2}$, and thus

$$
\left|k h(x)(x-a)^{k-1}\right| \leq k C_{2}|x-a|^{k} .
$$

Therefore, (42) can be written as

$$
g^{\prime}(x)=g^{\prime}(a)+g^{\prime \prime}(a)(x-a)+\cdots+\frac{g^{(k)}(a)}{(k-1) !}(x-a)^{k-1}+O(x-a)^{k} .
$$


Since the first, second, ..., and (k-1)-th derivatives of $g^{\prime}(x)$ at $a$ are $g^{\prime \prime}(a), g^{(3)}(a)$, $\ldots$, and $g^{(k)}(a)$, respectively, Equation (45) is the Taylor expansion of $g^{\prime}(x)$ at $a$ to order $k-1$.

Usually we write (40) as

$$
\begin{aligned}
g(x)=g(a)+ & g^{\prime}(a)(x-a) \\
& +\frac{g^{\prime \prime}(a)}{2 !}(x-a)^{2}+\cdots+\frac{g^{(k)}(a)}{k !}(x-a)^{k}+O\left((x-a)^{k+1}\right) .
\end{aligned}
$$

This shows that we can formally differentiate (46) to get (45).

\section{References}

[Agarwal and Lakshmikantham 1993] R. P. Agarwal and V. Lakshmikantham, Uniqueness and nonuniqueness criteria for ordinary differential equations, Series in Real Analysis 6, World Scientific Publishing, River Edge, NJ, 1993. MR 96e:34002 Zbl 0785.34003

[Li and Nirenberg 2006] Y. Y. Li and L. Nirenberg, "A geometric problem and the Hopf lemma, II", Chinese Ann. Math. Ser. B 27:2 (2006), 193-218. MR 2007i:53006 Zbl 1149.53302

[Pan and Wang 2008] Y. Pan and M. Wang, "When is a function not flat?", J. Math. Anal. Appl. 340:1 (2008), 536-542. MR 2008m:26048 Zbl 1131.26016

Received January 25, 2012. Revised February 20, 2012.

YIFEI PAN

Department of Mathematical SCiEnCES

INDIANA UNIVERSITY-PURDUE UNIVERSITY FORT WAYNE

FORT WAYNE, IN 46805

UNITED STATES

and

SCHOOL OF MATHEMATICS AND INFORMATICS

JIANGXI NORMAL UNIVERSITY

NANCHANG

CHINA

pan@ipfw.edu

MeI WANG

DEPARTMENT OF STATISTICS

UNIVERSITY OF CHICAGO

ChICAGo, IL 60637

UNITED STATES

meiwang@galton.uchicago.edu

YU YAN

Department of Mathematics ANd Computer SCIENCE HUNTINGTON UNIVERSITY

HUNTINGTON, IN 46750

UNITED STATES

yyan@huntington.edu 


\title{
PACIFIC JOURNAL OF MATHEMATICS
}

\author{
msp.org/pjm
}

Founded in 1951 by E. F. Beckenbach (1906-1982) and F. Wolf (1904-1989)

\section{EDITORS}

V. S. Varadarajan (Managing Editor)

Department of Mathematics

University of California

Los Angeles, CA 90095-1555

pacific@math.ucla.edu

Paul Balmer

Department of Mathematics

University of California

Los Angeles, CA 90095-1555

balmer@math.ucla.edu

Daryl Cooper

Department of Mathematics

University of California

Santa Barbara, CA 93106-3080 cooper@math.ucsb.edu

Jiang-Hua $\mathrm{Lu}$

Department of Mathematics

The University of Hong Kong

Pokfulam Rd., Hong Kong jhlu@maths.hku.hk
Don Blasius

Department of Mathematics University of California

Los Angeles, CA 90095-1555

blasius@math.ucla.edu

Robert Finn

Department of Mathematics Stanford University

Stanford, CA 94305-2125

finn@math.stanford.edu

Sorin Popa

Department of Mathematics

University of California

Los Angeles, CA 90095-1555

popa@math.ucla.edu

Paul Yang

Department of Mathematics

Princeton University

Princeton NJ 08544-1000

yang@math.princeton.edu

\section{PRODUCTION}

Silvio Levy, Scientific Editor, production@msp.org

\section{SUPPORTING INSTITUTIONS}

ACADEMIA SINICA, TAIPEI

CALIFORNIA INST. OF TECHNOLOGY

INST. DE MATEMÁTICA PURA E APLICADA

KEIO UNIVERSITY

MATH. SCIENCES RESEARCH INSTITUTE

NEW MEXICO STATE UNIV.

OREGON STATE UNIV.

\author{
STANFORD UNIVERSITY \\ UNIV. OF BRITISH COLUMBIA \\ UNIV. OF CALIFORNIA, BERKELEY \\ UNIV. OF CALIFORNIA, DAVIS \\ UNIV. OF CALIFORNIA, LOS ANGELES \\ UNIV. OF CALIFORNIA, RIVERSIDE \\ UNIV. OF CALIFORNIA, SAN DIEGO \\ UNIV. OF CALIF., SANTA BARBARA
}

\author{
Vyjayanthi Chari \\ Department of Mathematics \\ University of California \\ Riverside, CA 92521-0135 \\ chari@math.ucr.edu \\ Kefeng Liu \\ Department of Mathematics \\ University of California \\ Los Angeles, CA 90095-1555 \\ liu@math.ucla.edu \\ Jie Qing \\ Department of Mathematics \\ University of California \\ Santa Cruz, CA 95064 \\ qing@cats.ucsc.edu
}

These supporting institutions contribute to the cost of publication of this Journal, but they are not owners or publishers and have no responsibility for its contents or policies.

See inside back cover or msp.org/pjm for submission instructions.

The subscription price for 2013 is US \$400/year for the electronic version, and \$485/year for print and electronic.

Subscriptions, requests for back issues and changes of subscribers address should be sent to Pacific Journal of Mathematics, P.O. Box 4163, Berkeley, CA 94704-0163, U.S.A. The Pacific Journal of Mathematics is indexed by Mathematical Reviews, Zentralblatt MATH, PASCAL CNRS Index, Referativnyi Zhurnal, Current Mathematical Publications and the Science Citation Index.

The Pacific Journal of Mathematics (ISSN 0030-8730) at the University of California, c/o Department of Mathematics, 798 Evans Hall \#3840, Berkeley, CA 94720-3840, is published monthly except July and August. Periodical rate postage paid at Berkeley, CA 94704, and additional mailing offices. POSTMASTER: send address changes to Pacific Journal of Mathematics, P.O. Box 4163, Berkeley, CA 94704-0163.

PJM peer review and production are managed by EditFLOW ${ }^{\circledR}$ from Mathematical Sciences Publishers.

PUBLISHED BY

mathematical sciences publishers

nonprofit scientific publishing

http://msp.org/

(C) 2013 Mathematical Sciences Publishers 


\section{PACIFIC JOURNAL OF MATHEMATICS}

Volume $263 \quad$ No. $2 \quad$ June 2013

Realizations of $B C_{r}$-graded intersection matrix algebras with grading 257 subalgebras of type $B_{r}, r \geq 3$

SANDEEP BHARGAVA and YUN GAO

Stable flags, trivializations and regular connections

283

Elie COMPOINT and EDUARDo COREL

Elliptic aliquot cycles of fixed length

353

NATHAN JONES

Asymptotic $L^{4}$ norm of polynomials derived from characters

DANIEL J. KATZ

Degree-three spin Hurwitz numbers

JUNHO LEE

$\left(\mathbb{Z}_{2}\right)^{3}$-colorings and right-angled hyperbolic 3-manifolds

YOULIN LI and JiMING MA

Real closed separation theorems and applications to group algebras

Tim NETZER and ANDREAS THOM

Uniqueness theorem for ordinary differential equations with Hölder continuity

YifEI PAN, MEI WANG and Yu YAN

An analogue to the Witt identity

G. A. T. F. DA CostA and G. A. ZimmermanN

On the classification of stable solutions to biharmonic problems in large dimensions

Juncheng Wei, Xingwang Xu and Wen Yang 